\title{
Thrombectomy 24 hours after stroke: beyond DAWN
}

\author{
Shashvat M Desai, ${ }^{1}$ Diogo C Haussen, ${ }^{2}$ Amin Aghaebrahim, ${ }^{3}$ Alhamza R Al-Bayati, ${ }^{2}$ \\ Roberta Santos, ${ }^{3}$ Raul G Nogueira, ${ }^{2}$ Tudor G Jovin, ${ }^{1}$ Ashutosh P Jadhav ${ }^{1}$
}

${ }^{1}$ University of Pittsburgh Medical Center, Pittsburgh, Pennsylvania, USA ${ }^{2}$ Emory University/Marcus Stroke \& Neuroscience Center-Grady Memorial Hospital, Atlanta, Georgia, USA ${ }^{3}$ Lyerly Neurosurgery, Baptist Neurological Institute, Jacksonville, Florida, USA

\section{Correspondence to}

Dr Ashutosh P Jadhav, University of Pittsburgh, Pittsburgh, PA 15218, USA; jadhavap@upmc. edu

Received 10 March 2018 Revised 3 May 2018 Accepted 10 May 2018 Published Online First 28 May 2018
Check for updates

To cite: Desai SM

Haussen DC, Aghaebrahim A et al. J Neurolntervent Surg 2018;10:1039-1042.

\section{ABSTRACT}

Background and purpose The results of the DAWN trial support the benefit of thrombectomy in patients with anterior circulation large vessel occlusion (LVO) acute stroke presenting within 6-24hours from time last known well (TLKW). We sought to evaluate the characteristics and outcomes of patients who met DAWN criteria but underwent thrombectomy beyond 24 hours of TLKW.

Methods A retrospective review of endovascular thrombectomy databases at three comprehensive stroke centers was performed to identify all patients who received thrombectomy beyond 24 hours of TLKW and otherwise met the DAWN criteria. Baseline characteristics, efficacy, and safety outcomes were compared with patients in the DAWN trial intervention arm.

Results Twenty-one patients met the inclusion criteria. Rates of successful reperfusion (mTICI2b-3: 81\% vs $84 \%, P=0.72), 90$-day functional independence (modified Rankin Scale score $0-2,43 \%$ vs $48 \%$, $P=0.68)$, and symptomatic intracranial hemorrhage (5\% vs $6 \%, P=0.87$ ) were comparable across the two groups. Conclusion Thrombectomy appears to be safe and feasible in patients with acute ischemic stroke due to LVO meeting all DAWN trial criteria but treated beyond 24 hours of TLKW with outcomes comparable to patients in the DAWN trial intervention arm. Further studies are warranted to validate these findings.

\section{INTRODUCTION}

Endovascular thrombectomy (ET) has revolutionized the treatment of acute ischemic stroke due to large vessel occlusion (LVO). Multiple randomized controlled trials have established the superiority of ET over medical management for patients presenting in the early window. ${ }^{1-5}$ The DAWN trial (Diffusion-weighted imaging or CT perfusion assessment with clinical mismatch in the triage of wake up and late presenting strokes undergoing neurointervention with Trevo) ${ }^{6}$ and the DEFUSE-3 trial (Endovascular Therapy Following Imaging Evaluation for Ischemic Stroke 3$)^{7}$ have demonstrated that ET is very effective in carefully selected patients well beyond conventional time windows. These findings have shifted the traditional time window to tissue window-based patient selection for ET. ${ }^{8-10}$ Given the large treatment effect in the DAWN trial, we sought to assess the performance of ET in patients with acute ischemic stroke meeting the DAWN criteria but otherwise receiving ET beyond 24 hours of time last known well (TLKW).

\section{METHODS}

After local Institutional Review Board approval, we performed a retrospective review of prospectively maintained ET databases at three tertiary care medical centers in the USA (University of Pittsburgh Medical Center, Pittsburgh, 2011-2017, 16 patients; Emory University/Marcus Stroke \& Neuroscience Center-Grady Memorial Hospital, Atlanta, 2010-2017, 3 patients; and Lyerly Neurosurgery, Baptist Neurological Institute, Jacksonville, 2014-2018, 2 patients). Demographic and clinical data were collected by systematic chart review. Radiological data from non-contrast CT of the head, CT angiography, and/or MR angiography, CT perfusion and/or diffusion weighted imaging (DWI) using MRI at admission, post procedure, and before discharge were collected and analyzed. Imaging modalities used depended on institutional physician preference and resources.

\section{Patient selection}

Inclusion criteria included acute ischemic stroke treated with ET beyond 24 hours since TLKW (including wake up, witnessed and unwitnessed strokes) with intracranial internal carotid artery (ICA) or first segment of middle cerebral artery (MCA M1) occlusion with or without extracranial occlusion and presence of age-based mismatch between clinical severity of stroke and infarct core volume (age $<80$ years: National Institutes of Health Stroke Scale (NIHSS) score $\geq 10$ and infarct core volume $<31 \mathrm{~mL}$ or NIHSS score $\geq 20$ and infarct core volume $<51 \mathrm{~mL}$; age $\geq 80$ years: NIHSS score $\geq 10 \mathrm{~mL}$ and infarct core volume $<21 \mathrm{~mL}$ ). Additional DAWN trial criteria of baseline modified Rankin Scale (mRS) score 0-1 was also applied. Patients with more than one-third of the MCA territory involvement and/or hemorrhage on CT head were excluded. Site of occlusion was determined using CT angiography or MR angiography and ischemic core volumes were measured on CT perfusion or DWI-MRI using automated software RAPID (iSchemaView, Menlo Park, California, USA).

\section{End points}

The primary outcome was the rate of functional independence (defined as mRS score of 0,1 , or 2) at 90 days and was obtained from systematic review of follow-up visit, physical rehabilitation, and/ or mortality documentation. The secondary outcome included early therapeutic response (defined as a decrease in the NIHSS score $\geq 10$ from baseline or an NIHSS score of 0 or 1 on day 5,6 , or 7 of hospitalization or at discharge if it occurred before day 5). 
The primary safety outcome was all-cause 90-day mortality. Other safety outcomes were neurological deterioration (defined as an increase in NIHSS score of $\geq 4$ points within 5 days after stroke that was not attributed to intracranial hemorrhage $(\mathrm{ICH})$ or malignant cerebral edema) ${ }^{6}$ and symptomatic intracranial hemorrhage (sICH; defined according to the European Acute Stroke Study III criteria as the presence of extravascular blood in the cranium that was associated with an increase in the NIHSS score of $\geq 4$ points or death) ${ }^{11}$ within 24 hours after thrombectomy.

Procedural information, including device and technique used, as well as successful recanalization (defined as grade $2 \mathrm{~b}$ or 3 on the modified Thrombolysis in Cerebral Infarction (TICI) scale) was collected.

Baseline characteristics, efficacy outcomes, and safety and technical feasibility of treating patients beyond 24 hours since TLKW who met other DAWN criteria were analyzed and compared with patients in the DAWN trial intervention arm.

\section{Analysis of patients meeting DAWN criteria presenting beyond the 24 hour time window}

At the University of Pittsburgh Medical Center we analyzed the total number of patients screened during the DAWN trial enrollment period and identified the proportion of patients who would have met the DAWN criteria beyond 24 hours of TLKW.

\section{Statistical analysis}

Continuous variables are reported as mean \pm SD or median (IQR) as appropriate. Categorical variables are reported as proportions. Between-group comparison for continuous variables was conducted using the Student t-test and categorical variables using the $\chi^{2}$ test or Fisher exact test, as appropriate. Significance was defined as $\mathrm{P}<0.05$. Univariate, multivariate, and multivariable logistic regression analyses were performed. Statistical analysis was undertaken using IBM SPSS Statistics 23 (IBM-Armonk, New York, USA).

\section{RESULTS}

Twenty-one patients from three centers met the DAWN criteria and underwent ET more than 24 hours from TLKW. Individual patient data are shown in table 1. Baseline characteristics were comparable, with the exception of a significantly lower rate of atrial fibrillation and a higher frequency of unwitnessed stroke and intracranial carotid occlusions in the DAWN eligible group (table 2).

Procedural, efficacy, and safety outcomes were compared (table 3 ). Modified TICI $\geq 2$ b rates $(81 \%$ vs $84 \%, \mathrm{P}=0.72)$ and groin puncture to revascularization time (median 49.6 vs $48 \mathrm{~min}$ ) were comparable between the two groups. Good outcome (mRS $0-2$ at 90 days) was comparable between DAWN eligible patients and those in the DAWN intervention arm (43\% vs $48 \%, \mathrm{P}=0.68)$. There was no difference in rates of sICH (5\% vs $6 \%, \mathrm{P}=0.87)$ and mortality $(19 \%$ vs $19 \%, \mathrm{P}=0.96)$. ET was performed from just beyond 24 hours to up to 6 days from TLKW.

A review of the screening log at the University of Pittsburgh revealed that 16 patients presented with anterior circulation LVO during the DAWN trial enrollment period (November 2014 to February 2017). Of these, five patients underwent ET beyond 24 hours of TLKW and met all other DAWN criteria. These patients account for $0.2 \%$ of all acute ischemic stroke

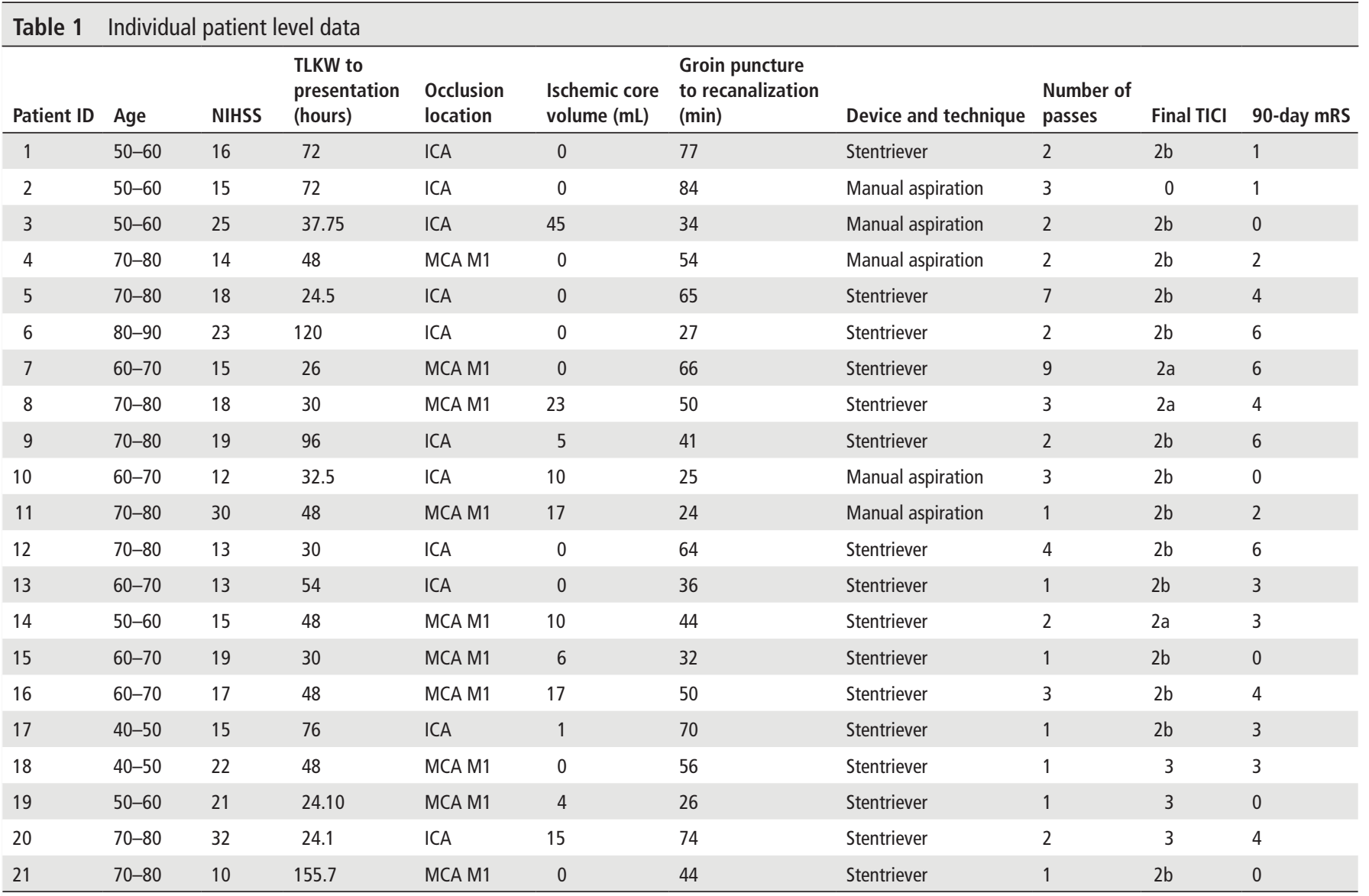

ICA, internal carotid artery; MCA M1, middle cerebral artery segment 1; mRS, modified Rankin Scale; NIHSS, National Institutes of Health Stroke Scale; TICI, Thrombolysis in Cerebral Infarction; TLKW, time last known well. 
Table 2 Baseline characteristics

\begin{tabular}{|c|c|c|c|}
\hline & $\begin{array}{l}\text { DAWN eligible ( }>24 \text { hours since TLKW) } \\
n=21\end{array}$ & $\begin{array}{l}\text { DAWN trial intervention arm } \\
\mathrm{n}=107\end{array}$ & $P$ values \\
\hline Age, mean $\pm S D$ & $65.2 \pm 11.1$ & $69.4 \pm 14.1$ & 0.2 \\
\hline Male sex, n (\%) & $8(38 \%)$ & $42(39 \%)$ & 0.66 \\
\hline Hypertension, n (\%) & $17(81 \%)$ & $83(78 \%)$ & 0.73 \\
\hline Diabetes mellitus, n (\%) & $7(33 \%)$ & $26(24 \%)$ & 0.38 \\
\hline Atrial fibrillation, $\mathrm{n}(\%)$ & $2(9.5 \%)$ & $43(40 \%)$ & 0.007 \\
\hline \multicolumn{4}{|l|}{ Presentation of stroke, $\mathrm{n}(\%)$} \\
\hline Wake up & $1(5 \%)$ & $67(63 \%)$ & \multirow[t]{3}{*}{$<0.0001$} \\
\hline Unwitnessed & $20(95 \%)$ & $29(27 \%)$ & \\
\hline Witnessed & $0(0 \%)$ & $11(10 \%)$ & \\
\hline Admission NIHSS score & $18.2 \pm 5.9$ & $17.4 \pm 5.1$ & 0.52 \\
\hline \multicolumn{4}{|l|}{ Occlusion location, n (\%) } \\
\hline Internal carotid artery & $11(52 \%)$ & $22(21 \%)$ & \multirow[t]{2}{*}{0.001} \\
\hline Middle cerebral artery & $10(48 \%)$ & $83(78 \%)$ & \\
\hline Ischemic core imaging study & $71 \%$ CTP, 29\% MRI-DWI & $63 \%$ CTP, 37\% MRI-DWI & 0.46 \\
\hline Ischemic core volume, $\mathrm{mL}$ & $7.3 \pm 11.2$ & $11.1 \pm 11.2$ & 0.15 \\
\hline TLKW to groin puncture, hours (median (IQR)) & $48(30-72)$ & $12.8(106-16.7)$ & N/A \\
\hline \multicolumn{4}{|l|}{ Device/technique. n (\%) } \\
\hline Manual aspiration only & $5(24 \%)$ & $0(0 \%)$ & \multirow[t]{2}{*}{$<0.0001$} \\
\hline Stentriever assisted & $16(76 \%)$ & $107(100 \%)$ & \\
\hline Number of passes & $2.5 \pm 2$ & $2.4 \pm 1.5$ & 0.79 \\
\hline
\end{tabular}

N/A, insufficient individual patient level data for DAWN trial to compare median (IQR) between the two groups.

admissions during the enrollment period $(n=2667)$. One-third of patients presenting with a severe stroke (NIHSS $\geq 10$ ) due to LVO met the DAWN criteria in the $>24$ hour time window (5 of 16).

\section{DISCUSSION}

The therapeutic time window for the treatment of acute ischemic stroke due to LVO using ET has expanded considerably. ${ }^{6-8}$ The DEFUSE-3 trial demonstrated benefit with mechanical thrombectomy up to 16 hours from TLKW and the DAWN trial enrolled patients up to 24 hours since LKW. Physiology-based patient selection by identifying clinical core mismatch (DAWN trial) or identification of a target mismatch on perfusion imaging (DEFUSE-3 trial) represents a shift from a strictly time-based

Table 3 Outcomes

\begin{tabular}{|c|c|c|c|}
\hline & $\begin{array}{l}\text { DAWN eligible } \\
\text { (>24 hours } \\
\text { since TLKW) } \\
n=21\end{array}$ & $\begin{array}{l}\text { DAWN trial } \\
\text { intervention } \\
\text { arm } n=107\end{array}$ & P values \\
\hline \multicolumn{4}{|l|}{ Procedural outcomes, n (\%) } \\
\hline Rates of $\mathrm{TICl} \geq 2 \mathrm{~b}$ & $17(81 \%)$ & $90(84 \%)$ & 0.72 \\
\hline \multicolumn{4}{|l|}{ Efficacy outcomes, n (\%) } \\
\hline Early neurological recovery & $6(29 \%)$ & $51(48 \%)$ & 0.10 \\
\hline mRS $0-2$ at 90 days & $9(43 \%)$ & $51(48 \%)$ & 0.68 \\
\hline \multicolumn{4}{|l|}{ Safety outcomes, n (\%) } \\
\hline Neurologic deterioration & $2(10 \%)$ & $15(14 \%)$ & 0.57 \\
\hline Symptomatic ICH & $1(5 \%)$ & $6(6 \%)$ & 0.87 \\
\hline Mortality & $4(19 \%)$ & $20(19 \%)$ & 0.96 \\
\hline
\end{tabular}

patient selection paradigm. The American Heart Association 2018 guidelines proposed class $1 \mathrm{~A}$ recommendation for thrombectomy in the 6-16hour window for patients meeting DAWN and/or DEFUSE-3 trial criteria and class IIA recommendation for patients meeting DAWN trial criteria in the $16-24$ hour time window. ${ }^{8}$

The results of the DAWN trial have shown that there exists a proportion of patients with LVO strokes who have enough collateral support to sustain a penumbra and harbor small ischemic cores ('slow progressors'). ${ }^{9}$ The median change in ischemic core volume at 24 hours in the DAWN control arm was $13 \mathrm{~mL}$ (median infarct growth rate of $0.5 \mathrm{~mL} /$ hour). ${ }^{6}$ Brisk enrollment in the DAWN trial and a recent retrospective study suggest that the proportion of such trial eligible patients is high. ${ }^{12}$ Second, the poor natural history of patients in the DAWN control arm (mRS $0-2$ at 90 days achieved in 13\% of patients) confirms that collaterals eventually fail and the ischemic core volume grows further. Third, revascularization is procedurally feasible (mTICI $\geq 2 \mathrm{~b}$ rates $84 \%$ ), safe (sICH rates $6 \%$, mortality rates $19 \%$ ) and in line with early time window thrombectomy trials.

To the best of our knowledge, this case series of 21 patients is the largest for anterior circulation thrombectomy beyond 24 hours of LKW. This series suggests that the benefit of ET persists beyond 24 hours when selecting patients using DAWN criteria (good outcome rate of 43\%) without compromising safety (low sICH rate of 5\%, mortality rate of 19\%).

With mTICI $\geq 2 \mathrm{~b}$ rates of $81 \%$, technical success in this series is similar to the DAWN trial intervention arm (84\%). It has been previously reported that successful recanalization is achievable with an acceptable safety profile in subacute ( $>48$ hours to 5 days) intracranial occlusions. ${ }^{13}$ In our series, patients were treated beyond 24 hours and up to 6 days of LKW.

We recently reported that $1.7 \%$ of all patients with acute ischemic stroke presenting to a comprehensive stroke center met all 
DAWN criteria. ${ }^{14}$ Hence, $1.9 \%$ (1.7\% DAWN eligible 6-24 hours ${ }^{14}$ plus $0.2 \%$ DAWN eligible $>24$ hours) of all acute ischemic strokes met DAWN criteria beyond 6 hours of TLKW. This represents an increase in thrombectomy eligibility and eventual reduction of disability due to LVO stroke.

DAWN and DEFUSE-3 trials have significant implications for stroke systems of care in the USA, and potentially even more so for the developing world. Simplifying physiology-based patient selection using non-contrast CT alone (without automated software processing $)^{15}$ will lead to wider implementation, reduction in door-to-needle times, and reduce expenditure associated with advanced imaging and processing.

Our study is limited by its retrospective nature and small sample size. Furthermore, a substantially lower proportion of patients in our series had atrial fibrillation compared with patients in the DAWN trial intervention arm and this may have a bearing on outcomes. We recognize that a fraction of 'unwitnessed strokes' may actually have a true stroke onset to presentation time of less than 24 hours, similar to the DAWN and DEFUSE-3 trials. However, our findings support the concept of directing patient selection on a tissue-based paradigm without any clear time cut-off, after which reperfusion ceases to be beneficial in appropriately selected patients. Nonetheless, benefit likely diminishes in a time-dependent fashion.

\section{CONCLUSION}

Our series suggests that ET appears to be safe and feasible in patients with acute ischemic stroke due to LVO meeting all DAWN trial criteria but treated beyond 24 hours of TLKW.

Contributors Conception and design: APJ. Acquisition of data: all authors. Analysis and interpretation of data: all authors. Drafting the article: SMD. Critically revising the article: all authors. Administrative/technical/material support: all authors. Study supervision: APJ.

Competing interests TGJ: Consultant: Neuravi (Steering Committee - modest), Codman Neurovascular (DSMB - modest), Stryker Neurovascular (PI DAWN - unpaid), Fundacio Ictus (PI REVASCAT - unpaid). Stock: Anaconda, Silk Road, Blockade Medical (modest). RGN: Stryker-Neurovacular (Trevo-2 \& DAWN Trial PI), Covidien (SWIFT \& SWIFT PRIME/Steering Committee, STAR Trial/ Core Lab), Penumbra (3-D Separator Trial/Executive Committee).
Patient consent Not required.

Ethics approval Ethics approval was obtained from the Institutional Review Boards of University of Pittsburgh, Emory University, and Lyerly Neurosurgery, Baptist Neurological Institute.

Provenance and peer review Not commissioned; externally peer reviewed.

(C) Article author(s) (or their employer(s) unless otherwise stated in the text of the article) 2018. All rights reserved. No commercial use is permitted unless otherwise expressly granted.

\section{REFERENCES}

1 Berkhemer OA, Fransen PS, Beumer D, et al. A randomized trial of intraarterial treatment for acute ischemic stroke. N Engl J Med 2015;372:11-20.

2 Goyal M, Demchuk AM, Menon BK, et al. Randomized assessment of rapid endovascular treatment of ischemic stroke. N Engl J Med 2015;372:1019-30.

3 Saver JL, Bonafe A, Bonafe A, et al. Stent-retriever thrombectomy after intravenous t-PA vs. t-PA alone in stroke. N Eng/ J Med 2015;372:2285-95.

4 Jovin TG, Chamorro A, Cobo E, et al. Thrombectomy within 8 hours after symptom onset in ischemic stroke. N Engl J Med 2015;372:2296-306.

5 Campbell BC, Mitchell PJ, Kleinig TJ, et al. EXTEND-IA Investigators. Endovascular therapy for ischemic stroke with perfusion-imaging selection. N Eng/ J Med 2015;372:1009-18.

6 Nogueira RG, Jadhav AP, Haussen DC, et al. Thrombectomy 6 to 24 hours after stroke with a mismatch between deficit and infarct. N Engl J Med 2018;378:11-21.

7 Albers GW, Marks MP, Kemp S, et al. Thrombectomy for stroke at 6 to 16 hours with selection by perfusion imaging. N Engl J Med 2018;378:708-18.

8 William J, Powers AA, Rabinstein TA, et al. 2018 Guidelines for the early management of patients with acute ischemic stroke: a guideline for healthcare professionals from the American Heart Association/American Stroke Association. Stroke 2018.

9 Rocha M, Jovin TG. Fast versus slow progressors of infarct growth in large vessel occlusion stroke. Stroke 2017:48:2621-7.

10 Albers GW. Late window paradox. Stroke 2018:49:768-71.

11 Hacke W, Kaste M, Bluhmki E, et al. ECASS Investigators. Thrombolysis with alteplase 3 to 4.5 hours after acute ischemic stroke. N Engl J Med 2008:359:1317-29.

12 Rocha M, Desai S, Jadhav A, et al. Abstract TP12: distribution and incidence of fast versus slow progressors of infarct growth in large vessel occlusion stroke. Stroke 2018;49.

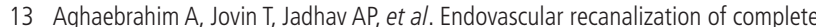
subacute to chronic atherosclerotic occlusions of intracranial arteries. J Neurointerv Surg 2014;6:645-8.

14 Jadhav AP, Desai SM, Kenmuir CL, et al. Eligibility for endovascular trial enrollment in the 6-24 hour time window: analysis of a single comprehensive stroke center. Stroke 20184910151017.

15 Bouslama M, Bowen MT, Haussen DC, et al. Selection paradigms for large vessel occlusion acute ischemic stroke endovascular therapy. Cerebrovasc Dis 2017;44:277-84 